\title{
Marrying medicine and materials: artemisinin (Qinghaosu) particle is soft enough for scratching hard SiC wafer in water
}

\author{
Yu-Rong Zhu' ${ }^{1}$, Dan Zhang ${ }^{1}$, Fei-Hu Zhang ${ }^{2}$, and Yang Gan*1 \\ ${ }^{1}$ School of Chemical Engineering and Technology, Harbin Institute of Technology, Harbin 150001, China \\ ${ }^{2}$ School of Mechanical Engineering, Harbin Institute of Technology, Harbin 150001, China \\ *Corresponding author's e-mail address: ygan@hit.edu.cn
}

Published online: 27 January 2016 (Version 1)

Cite as: Zhu et al. ScienceOpen Research 2016 (DOI: 10.14293/S2199-1006.1.SOR-MATSCI.AMNMZS.v1)

Reviewing status: Please note that this article is under continuous review. For the current reviewing status and the latest referee's comments please click here or scan the QR code at the end of this article.

Primary discipline: Materials science

Secondary discipline: Medicine, Chemistry, Engineering, Physics

Keywords SiC, Artemisinin, Chemical Mechanical Polishing, Hydroxyl Free Radical, Oxidation, Atomic Force Microscopy

\begin{abstract}
Silicon carbide $(\mathrm{SiC})$ single crystals, along with sapphire and silicon, are one of the most important substrates for highbrightness light-emitting diode fabrications. Owing to extremely high hardness (Mohs' scale of 9.5) and chemical inertness, the polishing rate of $\mathrm{SiC}$ with conventional chemical mechanical polishing methods is not high, and surface scratches are also inevitable because of using slurry containing hard abrasives such as silica particles. Here artemisinin (Qinghaosu) crystals, very soft molecular solids, were found, for the first time to the best of our knowledge, to effectively polish $\mathrm{SiC}$ wafers even in pure water as demonstrated by proof-of-concept scratching experiments using atomic force microscopy. The underlying mechanism is attributed to activated oxidation of $\mathrm{SiC}$ by mechanically released reactive $\cdot \mathrm{OH}$ free radicals from the endoperoxide bridges. The preliminary results reported here have important implications for developing novel alternative green and scratch-free polishing methods for hard-brittle substrates including $\mathrm{SiC}$, diamond, and others.
\end{abstract}

\section{INTRODUCTION}

Inventors of high-brightness blue light-emitting diode (LED) were awarded the 2014 Nobel prize for physics [1]. Silicon carbide ( $\mathrm{SiC}$ ) single crystals, along with sapphire, GaN, and silicon, are one of the most important substrates for highbrightness LED fabrications. Among all processes leading to final LED devices such as crystal growth, slicing, polishing, deposition of functional layers, and packaging, chemical mechanical polishing (CMP) is one of the key steps enabling successful deposition of functional III-V group layers such as GaN (as well as buffer layers).

With the CMP process, pre-polished SiC wafers, with surface roughness at nanometers scale, are subjected to simultaneous chemical and mechanical action in a CMP machine where a loaded substrate is rotated against a polishing pad with slurry continuously feed containing polishing colloids and various chemicals [2-4]. The final surface roughness after CMP is usually less than $0.5 \mathrm{~nm}$, thus enabling an atomic-scale smoothness to be achieved [5]. However, owing to the extremely high hardness (Mohs' scale of 9.5) and chemical inertness, the polishing rate of $\mathrm{SiC}$ with conventional CMP methods $[6,7]$ using slurry containing silica alumina or ceria colloids under harsh alkaline conditions is low [8-11]. At the same time, surface scratches are inevitable owing to a rather high hardness of polishing colloid particles.

Recently, oxidative solutions such as hydrogen peroxide have been introduced into SiC CMP slurry to explore the possibility of accelerated oxidation and removal, taking advantage of highly reactive $\mathrm{OH}$ hydroxyl free radicals released from hydrogen peroxide molecules $[8,13,14]$. Nevertheless, regardless of progress, the problems of persistent scratches and treating/recycling large amounts of used harmful wastes have yet to be solved. A new method for green, scratch-free CMP processing of SiC substrates is thus highly desired.

Discovery of artemisinin (Qinghaosu) as a revolutionary antimalarial drug fetched Youyou Tu the 2015 Nobel prize for physiology or medicine [15]. Artemisinin, with a molecular formula of $\mathrm{C}_{15} \mathrm{H}_{22} \mathrm{O}_{5}$ and with a molecular weight of $282 \mathrm{Da}$, belongs to sesquiterpene lactone endoperoxide. Researchers generally believe that it is the unique endoperoxide bridges that make artemisinin very effective in curing malaria through iron-mediated release of $\cdot \mathrm{OH}$ free radicals after cleaving endoperoxide bridges [16]. However, unfortunately this special Fenton-like reaction has not been paid due attention outside the pharmaceutical area.

In this study, two seemingly alien areas - artemisinin and polishing - were combined. Soft artemisinin (Qinghaosu) crystals were surprisingly found to effectively polish SiC single crystals even in water as revealed by proof-of-concept scratching experiments using atomic force microscopy (AFM)-based 
colloidal probe nanotribology strategy $[17,18]$. The preliminary results reported here will have important implications for developing alternative green and scratch-free polishing methods for hard-brittle substrates.

\section{EXPERIMENTAL}

\section{Artemisinin (Qinghaosu) crystals, SiC wafers}

Epitaxial (EPI) grade polished single-crystal c-plane $6 \mathrm{H}-\mathrm{SiC}$ single crystal substrates were $10 \mathrm{~mm} \times 10 \mathrm{~mm} \times 0.2 \mathrm{~mm}$ in size (Hefei Ke Jing Materials Technology Co., China). Commercial artemisinin (Qinghaosu) crystals $\left(\mathrm{C}_{15} \mathrm{H}_{22} \mathrm{O}_{5}\right.$, CAS No. 6396864-9) were produced from the extract of leaf Artemisia annua L with the batch number of SWX2015082504 (Xi'an Zebang Biological Technology Co., China). The purity specified by the manufacturer is $99.2 \%$ as examined by the high performance liquid chromatography (HPLC) method. The crystals, white needle-like, $20-100 \mu \mathrm{m}$ in width and 50-500 $\mu \mathrm{m}$ in length as examined with an optical microscopy, were used as-received without further purification.

\section{Wet cleaning of SiC wafers}

$\mathrm{SiC}$ wafers were first immersed and sonicated in the diluted detergent solution (15PF-x, Deconex Co., Germany) for $40 \mathrm{~min}$, rinsed with copious de-ionized (DI) water (resistivity of 18.2 $\mathrm{M} / \mathrm{cm})$, then soaked in the piranha solution $\left(\mathrm{H}_{2} \mathrm{SO}_{4}+\mathrm{H}_{2} \mathrm{O}_{2} 3: 1\right.$ mixture) for $30 \mathrm{~min}$ at $100^{\circ} \mathrm{C}$ (Caution: This Solution is Dangerous. Handle with Care!), finally rinsed with copious DI water and blown dry with a stream of high-speed nitrogen ( $\geq 99.99 \%$ ) using a nitrogen gun (KITZ, Japan). Notably, the SiC substrates were easier to be critically cleaned than sapphire substrates $[19,20]$. To avoid silica contamination, polyfluortetraethylene (PTFE) plastic wares were used for each wet cleaning step. Cleaned $\mathrm{SiC}$ substrates were stored in a sealed PTFE container prior to tests.

\section{AFM characterization}

A MultiMode 8 AFM system (Bruker Co., USA) including a NanoScope $\mathrm{V}$ controller was used in contact mode for scratching tests in water, and in the tapping/ScanAsys mode for topographical characterization. The scanner (model AS130VLR) is capable of $125 \mu \mathrm{m} \times 125 \mu \mathrm{m} \times 5 \mu \mathrm{m}$ scan range. All topographical images were captured and saved in the raw format and processed with the free WSXM software [21]. Silicon probes (NSG10, NT-MDT Co., Russia) with a nominal spring constant of $11.5 \mathrm{~N} / \mathrm{m}$ were used for AFM topographical characterization.

\section{Preparation of artemisinin-modified AFM probe}

Tipless silicon probes (All-In-One-TL, Budget Sensors, Bulgaria) with nominal spring constant of $40 \mathrm{~N} / \mathrm{m}$ were used for preparation of artemisinin modified probe and AFM scratching tests. Artemisinin-modified probes were prepared by gluing, with UV-curable glue (Norland Products Inc., USA), a small artemisinin crystal (or a broken part of larger crystals) of tens of micrometers in size to the cantilever of the tipless probe following the cantilever-moving procedure [22]. Curing of glue was carried out in a home-built UV light unit containing a UV light lamp (UVGL-55, UVP Inc, USA) fixed in a stainless steel container with a cover lid. At the same time, UV light irradiation also cleaned the probe by removing organic contaminants.

\section{AFM scratching test using artemisinin-modified AFM probe in water}

The procedure for a typical AFM scratching test is as follows. First, the artemisinin probe was examined by a microscope to ensure the artemisinin crystals were successfully attached to the cantilever. Then the probe was engaged with the substrate in water (with a quartz liquid cell) to scratch at a constant loading force $(10.3 \mu \mathrm{N}$, the maximum force executed by the cantilever used) over a $5 \mu \mathrm{m} \times 5 \mu \mathrm{m}$ area at $1 \mathrm{~Hz}$ for $60 \mathrm{~min}$ without break. After disengagement, the substrate was taken out and cleaned with the same cleaning procedure above, and the scratched area was subjected to AFM imaging for topographical analysis. The $\mathrm{pH}$ of water was adjusted using
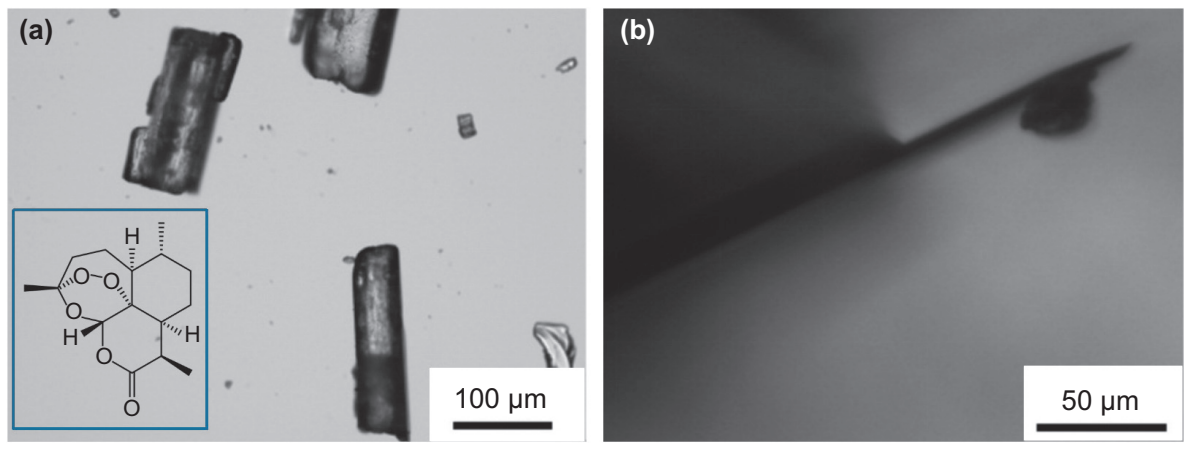

Figure 1. (a) Optical micrograph of several artemisinin crystals spread on glass slides. The inset shows the molecular structure of artemisinin. (b) Optical microscopy side view of a tipless AFM cantilever with an irregular shaped artemisinin crystal glued close to the triangular end of the cantilever. 
$\mathrm{NaOH}$ and $\mathrm{H}_{2} \mathrm{SO}_{4}$. Analytical grade chemical reagents were used. The temperature of the AFM laboratory was around $25^{\circ} \mathrm{C}$ and relative humidity was around $20 \%$.

\section{RESULTS AND DISCUSSION}

\section{Artemisinin crystals, artemisinin-modified AFM probe}

Optical micrograph of a few artemisinin crystals spread on glass slides, as shown in Figure 1a, shows varying size of artemisinin crystals with width from 50 to $100 \mu \mathrm{m}$. The crystal glued to the AFM cantilever was generally smaller than $50 \mu \mathrm{m}$, i.e., either a crystal smaller than those shown in Figure 1a or a broken part of a larger crystals was used. The artemisinin probe was examined by a microscope before AFM test, Figure $1 \mathrm{~b}$ shows the side view of a tipless AFM cantilever with an irregular shaped artemisinin crystal successfully glued close to the triangular end of the cantilever. This type of artemisinin crystal-modified AFM probe was used for AFM scratching test in water with a liquid cell. Note that pharmaceutical researchers used the similar drug particle-modified AFM probes, the socalled colloidal drug probes by some, for adhesion studies [23-25].

\section{AFM characterization of scratched substrates}

AFM topographical image of SiC substrates after being scratched with the artemisinin-modified probe for $60 \mathrm{~min}$ in DI water was recorded after the substrate was disengaged with the probe and cleaned. As shown in Figure 2a, even the scratching area was set to as small as $5 \mu \mathrm{m} \times 5 \mu \mathrm{m}$ for this test, multiple squares (four of them) could be found. This was attributed to either simultaneous multiple contacts between irregularly shaped artemisinin crystal and the substrate, or to varying contact asperities caused by gradual wearing of the probe against the substrate during scratching as observed for silica spheres [17]. Notably, besides these scratched areas, a straight scratch line running from the bottom to the top of the image as marked by two white arrows, about $1 \mu \mathrm{m}$ in width, disappeared within the scratched area. Disappearance of this scratch line which existed in the substrate before the test strongly indicates a removal of substrate materials, and this is clear from the three-dimensionally rendered topographical image (Figure $2 \mathrm{~b}$ ). Line profiles along two lines, right to each other across the scratched area, indicating the maximum depth was $\sim 0.5 \mathrm{~nm}$ for the deepest area, whereas some parts were shallower.

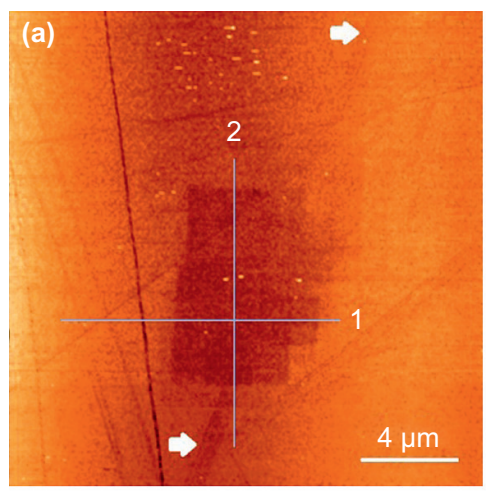

(b)

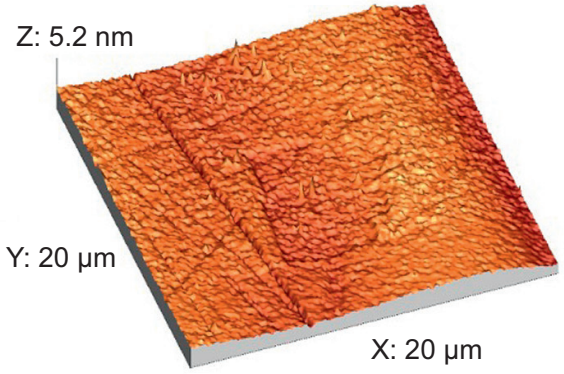

(c)
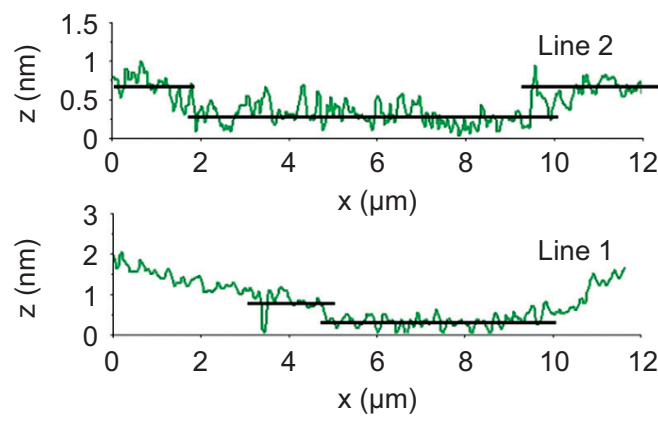

Figure 2. AFM topographical characterization of SiC substrates after scratched with the artemisinin crystal-modified probe shown above for $\mathbf{6 0} \mathrm{min}$ in DI water. (a) The two-dimensional topographical image. The image was only first-order flattened without other filtering treatments. The scratching area was $5 \mu \mathrm{m} \times 5 \mu \mathrm{m}$ for this case. Apparent multiple squares (four of them) were visible. Numbers 1 and 2 indicate two lines for line profile analyses shown in (c). Two arrows in the image draw the attention to a straight scratch line that disappeared within the scratched area. (b) Three-dimensional rendering of the topographical image, the scratched areas were clearly visible. (c) Line profiles along two lines 1 and 2 marked in (a), indicating a maximum depth of $0.5 \mathrm{~nm}$ of the scratched area. Note the bow of the image appeared in (b) and line profile for line 1 was an artifact caused by image-flattening operation with the software. 


\section{Comparing hardness of artemisinin with SiC and other common abrasives}

It is surprising, from traditional CMP process and materials point of view, that very hard SiC with Mohs' scale of 9.5 can be scratched by a much softer organic molecular crystal such as artemisinin. The quantitative values of hardness of artemisinin are not available both experimentally and theoretically at the moment to the best of our knowledge. We can have the order of magnitude estimate of the relative hardness of SiC, quartz, and artemisinin by comparing their melting point which is closely related to the strength of cohesive bonds: $\mathrm{SiC}\left(2,730^{\circ} \mathrm{C}\right.$, a covalent crystal), quartz $\left(1,750^{\circ} \mathrm{C}\right.$, a covalent and ionic crystal), and artemisinin $\left(152^{\circ} \mathrm{C}\right.$, a van der Waals crystal). We can thus safely expect very low hardness of artemisinin crystals - much lower than all common polishing solids such as silica, alumina, a ceria. The hardness of artemisinin may be comparable with other pharmaceutical solids such as ibuprofen [26]. The reasoning about very low hardness of artemisinin thus leads us naturally to conclude that it is impossible to explain the scratching results from a pure mechanical point of view.

\section{Mechanism discussion: decomposition of SiC mediated by mechanically activated $\mathrm{OH}$ free radicals in water}

Therefore, we need to turn to the intrinsic chemical nature of artemisinin to interpret the observed results. We noticed the fact that it is the unique endoperoxide bridge in a artemisinin molecule that makes artemisinin very effective in curing malaria by iron-mediated release of $\cdot 0 \mathrm{H}$ free radicals through cleaving endoperoxide bridges [16]. However, this special Fenton-like reaction has not been paid due attention outside pharmaceutical areas. It thus occurs to us that the underlying polishing mechanism can be reasonably attributed to activated oxidation and the subsequent removal of $\mathrm{SiC}$ substrate materials mediated by the release of highly reactive $\cdot \mathrm{OH}$ free radicals from the endoperoxide bonds under mechanical activation, following the proposed reactions (1) and (2), as illustrated during the AFM scratching test (Figure $3 a$ and $b$ ):

$$
\begin{gathered}
-\mathrm{O}-\mathrm{O}-+2 \mathrm{H}_{2} \mathrm{O} \rightarrow 4 \mathrm{OH} \bullet \text { (activated mechanically) } \\
2 \mathrm{SiC}+4 \mathrm{OH} \bullet+3 \mathrm{O}_{2} \rightarrow 2 \mathrm{SiO}_{3}^{2-}+4 \mathrm{H}^{+}+2 \mathrm{CO}_{2}
\end{gathered}
$$

The proposed reaction (2) is not involved with the generation of $\mathrm{SiO}_{2}$, as commonly proposed by others in $\mathrm{H}_{2} \mathrm{O}_{2}$-mediated $\mathrm{SiC}$ CMP $[13,14]$ where silica particles are capable of mechanically removing $\mathrm{SiO}_{2}$ because artemisinin crystal is too soft to allow a mechanical removal of harder $\mathrm{SiO}_{2}$. Therefore, the proposed reactions favor the removal of $\mathrm{SiC}$ through direct decomposition of $\mathrm{SiC}$ into soluble species $\mathrm{SiO}_{3}{ }^{2-}$ instead of solid $\mathrm{SiO}_{2}$. Although the reaction (2) tells that higher $\mathrm{pH}$ will facilitate the removal of $\mathrm{SiC}$, our preliminary experiments carried out in

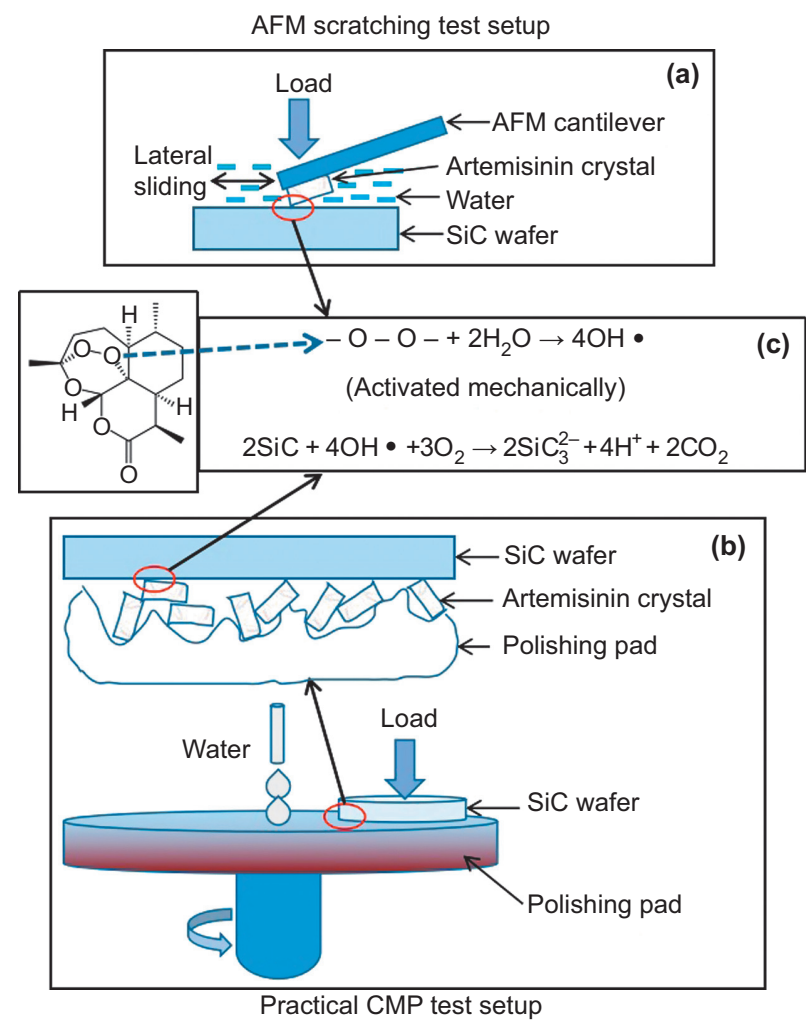

Figure 3. Proposed mechanism for artemisinin-mediated chemical mechanical polishing of SiC wafers in water. Both (a) the AFM scratching setup used in this study and (b) a practical CMP setup (to be investigated in future) with a rotating polishing pad and a loaded SiC wafer were illustrated. (c) Highly reactive $\cdot \mathrm{OH}$ free radicals were released from endoperoxide bonds under mechanical activation to facilitate direct decomposition of SiC into $\mathrm{CO}_{2}$ and soluble species $\mathrm{SiO}_{3}{ }^{2-}$. Red circles with arrows in (a) and (b) direct to magnified cartoons or the reaction mechanism scheme.

different $\mathrm{pH}$ conditions (results not shown here) show that acidic or neutral conditions, rather than basic conditions, favor the removal of SiC. But it should be kept in mind that the experimental $\mathrm{pH}$ dependence must be taken with caution because of significantly varying particle shape and scratching contact geometry among different tests. Here we only speculate that $\mathrm{pH}$ dependence, if confirmed by future results, may correlate with reaction (1) or stability of artemisinin. Clearly, the detailed mechanistic pathway, together with practical CMP tests (Figure 3c), needs a careful and systematic study.

\section{Possible strategy for increasing polishing rate}

One may argue that a very low removal rate $(0.5 \mathrm{~nm} / \mathrm{h})$ and low hardness of artemisinin crystals might render it an impractical candidate for practical CMP of hard materials such as SiC. It is true that, as discussed above, low hardness is intrinsic to molecular solids such as artemisinin, thus leading to a larger contact area, reduced local contact pressure, and 
fracture during scratching. Therefore, the low removal rate is not unexpected. Frankly speaking, we indeed found that artemisinin crystals attached to the AFM probe wore away markedly after the 60-min scratching test, indicating low hardness compared with silica particles for CMP [17]. However, there are ways to bypass this problem, for example, by mixing artemisinin with other polishing materials such as silica or iron to produce composite particles or the so-called hybrid particles [27]. In this way, artemisinin crystals may act as agents for releasing $\cdot \mathrm{OH}$ free radicals and other materials can take the role of mechanical abrasives and/or Fenton reaction-type activators (for the case of iron).

\section{CONCLUSIONS}

Artemisinin (Qinghaosu) crystalline particles were successfully used, for the first time to the best of our knowledge, to effectively polish $\mathrm{SiC}$ single crystals even in water as revealed by proof-of-concept scratching experiments using AFM. A material removal rate of $\sim 0.5 \mathrm{~nm} / \mathrm{h}$ was achieved using the irregular-shaped artemisinin particles (tens of micrometers in size) under a load of $\sim 10 \mu \mathrm{N}$. The underlying mechanism is attributed to activated oxidation and subsequent removal of substrate materials owing to the release of highly reactive $\cdot \mathrm{OH}$ free radicals from the endoperoxide bonds under mechanical activation. The preliminary results reported here will have important implications for developing novel alternative green and scratch-free CMP methods for hard-brittle substrates including $\mathrm{SiC}$, diamond, and others.

\section{Acknowledgements}

This project was sponsored by the National Basic Research Program of China under grant No. 2011CB013200, National Natural Science Foundation under grant No. 51535003, and Interdisciplinary Basic Research Fund of Science-EngineeringMedicine in HIT (Harbin Institute of Technology).

\section{References}

[1] Nakamura S, Pearton S, Fasol G. The Blue Laser Diode: the complete story. 2nd Ed. New York: Springer-Verlag; 2000.

[2] Zantye PB, Kumar A, Sikder AK. Chemical mechanical planarization for microelectronics applications. Mater Sci Eng R-Rep. 2004;45:89-220. doi:10.1016/j.mser.2004.06.002.

[3] Luo J, Dornfeld DA. Material removal mechanism in chemical mechanical polishing: theory and modeling. IEEE Trans Semicond Manuf. 2000;14:112-133.

[4] Kasai T, Bhushan B. Physics and tribology of chemical mechanical planarization. J Phys Condens Matter. 2008;20:225011. doi:10.1088/0953-8984/20/22/225011.

[5] Aida H, Doi T, Takeda H, Katakura H, Kim S-W, Koyama K, Yamazaki T, Uneda M. Ultraprecision CMP for sapphire, GaN, and $\mathrm{SiC}$ for advanced optoelectronics materials. Curr Appl Phys. 2012;12:41-46. doi:10.1016/j.cap.2012.02.016.

[6] Gutsche HW. Preparation of damage-free surface on alphaalumina. US Patent, 4011099. 1977-03-08.

[7] Gutsche HW, Moody JW. Polishing of sapphire with colloidal silica polishing. J Electrochem Soc. 1978;125:136-138. doi:10. 1149/1.2131378.
[8] Deng H, Endo K, Yamamura K. Competition between surface modification and abrasive polishing: a method of controlling the surface atomic structure of 4H-SiC (0001). Sci Rep. 2015;5:8947. doi:10.1038/srep08947.

[9] Pan G, Zhou Y, Luo G, Shi X, Zou C, Gong H. Chemical mechanical polishing (CMP) of on-axis Si-face $6 \mathrm{H}-\mathrm{SiC}$ wafer for obtaining atomically flat defect-free surface. J Mater Sci Mater Electron. 2013;24:5040-5047. doi:10.1007/s10854-013-1519-1.

[10] Shi X, Pan G, Zhou Y, Zou C, Gong H. Extended study of the atomic step-terrace structure on hexagonal $\mathrm{SiC}$ (0001) by chemical-mechanical planarization. Appl Surf Sci. 2013;284: 195-206. doi:10.1016/j.apsusc.2013.07.080.

[11] Zhou Y, Pan G, Shi X, Zhang S, Gong H, Luo G. Effects of ultrasmooth surface atomic step morphology on chemical mechanical polishing (CMP) performances of sapphire and SiC wafers. Tribol Int. 2015;87:145-150. doi:10.1016/j.triboint.2015.02.013.

[12] Zhou Y, Pan G, Shi X, Xua L, Zou C, Gong H, Luo G. XPS, UV-vis spectroscopy and AFM studies on removal mechanisms of Siface SiC wafer chemical mechanical polishing (CMP). Appl Surf Sci. 2014;316:643-648. doi:10.1016/j.apsusc.2014.08.011.

[13] Yagi K, Murata J, Kubota A, Sano Y, Hara H, Okamoto T, Arima K, Mimura H, Yamauchi K. Catalyst-referred etching of $4 \mathrm{H}-\mathrm{SiC}$ substrate utilizing hydroxyl radicals generated from hydrogen peroxide molecules. Surf Interface Anal. 2008;40:998-1001. doi:10.1002/sia.2804.

[14] Zhou Y, Pan G, Shi X, Gong H, Luo G, Gu Z. Chemical mechanical planarization (CMP) of on-axis Si-face SiC wafer using catalyst nanoparticles in slurry. Surf Coatings Technol. 2014;251:48-55. doi:10.1016/j.surfcoat.2014.03.044.

[15] Tu YY. The discovery of artemisinin (Qinghaosu) and gifts from Chinese medicine. Nat Med. 2011:17:1217-1220. doi:10.1038/ $\mathrm{nm} .2471$.

[16] Li J, Zhou B. Biological Actions of artemisinin: insights from medicinal chemistry studies. Molecules. 2010;5:1378-1397. doi:10.3390/molecules15031378.

[17] Zhang D, Chen X, Wang Y, Zhang F, Gan Y. Relationship between asperity-mediated surface forces and topography alteration of silica microspheres sliding on mica, sapphire, and glass substrates under ambient conditions: atomic force microscopy and theoretical studies. Langmuir. 2014;30:3729-3740. doi:10.1021/ la500355f.

[18] Miyoshi A, Nakagawa H, Matsukawa K. Simulation on chemical mechanical polishing using atomic force microscope. Microsystem Technol. 2005;11:1102-1106. doi:10.1007/s00542-0050509-5.

[19] Zhang D, Wang Y, Gan Y. Characterization of critically cleaned sapphire single-crystal substrates by atomic force microscopy, XPS and contact angle measurements. Appl Surf Sci. 2013;274: 405-417. doi:10.1016/j.apsusc.2012.12.143.

[20] Zhang D, Gan Y. Recent progress on cleaning of sapphire singlecrystal substrates: a mini-review. Recent Patents Chem Eng. 2013;6;161-166. doi:10.2174/2211334707999140331121752.

[21] Horcas I, Fernandez R, Gomez-Rodriguez J, Colchero J, GómezHerrero J, Baro AM. WSXM: a software for scanning probe microscopy and a tool for nanotechnology. Rev Sci Instrum. 2007;78:013705. doi:10.1063/1.2432410.

[22] Gan Y. A review of techniques for attaching micro- and nanoparticle to a probe's tip for surface force and near-field optical measurements. Rev Sci Instrum. 2007;78:081101. doi: 10.1063/1.2754076.

[23] Eve JK, Patel N, Luk SY, Ebbens SJ, Roberts CJ. A study of single drug particle adhesion interactions using atomic force microscopy. Int J Pharm. 2002;238:17-27. doi:10.1016/S0378-5173 (02)00055-8.

[24] Tsukada M, Irie R, Yonemochi Y, Noda R, Kamiya H, Watanabe W, Kauppinen EI. Adhesion force measurement of a 
DPI size pharmaceutical particle by colloid probe atomic force microscopy. Powder Technol. 2004;141:262-269. doi:10.1016/j. powtec.2004.03.008.

[25] Islam N, Tuli RA, George GA, Dargaville TR. Colloidal drug probe: method development and validation for adhesion force measurement using atomic force microscopy. Adv Powder Technol. 2014;25:1240-1248. doi:10.1016/j.apt.2014.06.021.

[26] Roberts RJ, Rowe RC, York P. The relationship between indentation hardness of organic solids and their molecular structure. J Mater Sci. 1994;29:2289-2296. doi:10.1007/ BF00363416.

[27] Lee HS, Kim DI, An JH, Lee HJ, Kim KH, Jeong H. Hybrid polishing mechanism of single crystal SiC using mixed abrasive slurry (MAS). CIRP Ann - Manuf Technol. 2010;59:333-336. doi:10.1016/j.cirp.2010.03.114.

\section{Competing Interests}

The authors declare no competing interests

\section{PUBLISHING NOTES}

(C) 2016 Zhu et al. This work has been published open access under Creative Commons Attribution License CC BY 4.0, which permits unrestricted use, distribution, and reproduction in any medium, provided the original work is properly cited Conditions, terms of use and publishing policy can be found at www.scienceopen.com.

Please note that this article may not have been peer reviewed yet and is under continuous post-publication peer review. For the current reviewing status please click here or scan the QR code on the right.

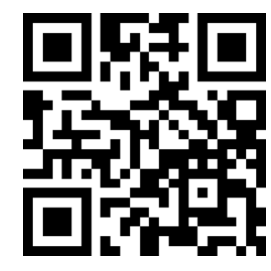

\section{ScienceOPEN.com}

